Pratiques informationnelles : Questions de modèles et de méthodes

\title{
Pratiques informationnelles et analyse des traces numériques : de la représentation à l'intervention
}

Information Practices and the Analysis of Digital Traces: From Representation to Intervention

\section{Bernhard Rieder}

\section{OpenEdition \\ Journals}

Édition électronique

URL : http://journals.openedition.org/edc/2249

DOI : 10.4000/edc.2249

ISSN : 2101-0366

Éditeur

Université Lille-3

\section{Édition imprimée}

Date de publication : 1 décembre 2010

Pagination : 91-104

ISBN : 978-2-917562-04-8

ISSN : 1270-6841

Référence électronique

Bernhard Rieder, «Pratiques informationnelles et analyse des traces numériques : de la représentation à l'intervention », Études de communication [En ligne], 35 | 2010, mis en ligne le 01 décembre 2012, consulté le 19 avril 2019. URL : http://journals.openedition.org/edc/2249; DOI 10.4000/edc. 2249

Ce document a été généré automatiquement le 19 avril 2019

(C) Tous droits réservés 


\title{
Pratiques informationnelles et analyse des traces numériques : de la représentation à l'intervention
}

\author{
Information Practices and the Analysis of Digital Traces: From Representation to \\ Intervention
}

Bernhard Rieder

1 Quand Gabriel Tarde écrivait en 1890 qu'il « pourra venir un moment où, de chaque fait social en train de s'accomplir, il s'échappera pour ainsi dire automatiquement un chiffre, lequel ira immédiatement prendre son rang sur les registres de la statistique " (Tarde, 1890, 192), le sociologue iconoclaste imaginait une situation qui parait, cent vingt ans après, en train de se réaliser. Dans les espaces numériques, qui ne cessent de se multiplier, chaque opération d'un utilisateur peut potentiellement déclencher la production d'une trace. Une requête sur un moteur de recherche, un échange sur messagerie instantanée, une déclaration sur un site de réseautage social, un mouvement de la souris, une hésitation avant la sélection du prochain lien - tous les gestes, aussi infimes soient-ils, peuvent être captés, enregistrés dans des fichiers de log ou bases de données et analysés par le biais de méthodes statistiques et autres.

2 Certaines de ces données sont rendues publiques, d'autres s'accumulent sans jamais être regardées, mais depuis quelques années l'intérêt que portent différents acteurs à ces " résidus " numériques ne cesse de croître. Pour les entreprises, il s'agit de comprendre des marchés traversés par un vacillement agité de tendances et composés d'ensembles de consommateurs se diversifiant progressivement en niches et sous niches. Les acteurs politiques espèrent prendre le pouls de sociétés emportées par une transformation de leurs modes d'organisation, leurs habitudes et leurs valeurs, basculant le paysage des luttes et des alliances politiques. Les chercheurs en Sciences Humaines et Sociales (SHS) finalement voient émerger devant leurs yeux un dispositif d'observation 
" total " qui promet de révolutionner les méthodologies classiques en effaçant le clivage entre micro et macro, entre qualitatif et quantitatif. La quantité et la richesse des données laissent penser que l'on pourrait passer, par une sorte de "zoom ", de l'ensemble à l'individu et de la moyenne à l'idiosyncrasie.

Dans tous ces cas on pense et espère que les traces dont les pratiques des internautes provoquent la production - précision nécessaire car l'enregistrement, le " logging ", n'a rien de " naturel ", il est conçu et construit pour atteindre des objectifs particuliers - " parlent vrai » sur la réalité sociale observée. Cette étonnante " ombre informationnelle » (O'Reilly et Battelle, 2009) se déployant derrière chaque usager est vue comme une source de connaissance, comme une représentation potentiellement authentique de groupes et d'individus, de leurs comportements, idées, besoins, valeurs, relations, antagonismes et ainsi de suite. Après les travaux pionniers de Govcom.org aux Pays-Bas ou alors de l'équipe WebAtlas en France un nombre très important de projets explore désormais les potentialités des " méthodes numériques " pour la recherche en SHS ; dans le marketing ce type d'analyse fait désormais partie de l'outillage de base.

Or les traces informationnelles forment non seulement des terrains d'analyse mais, à travers des boucles de feed-back, elles peuvent directement, et parfois en temps réel, contribuer au paramétrage des formes et fonctions proposées par un système informatique. Pour reprendre - avec un léger décalage - les termes d'Ian Hacking (1983), elles jouent un rôle aussi bien dans la représentation d'une réalité que dans l' intervention sur cette réalité. Les systèmes de personnalisation peuvent servir d'exemple : les achats qu'un consommateur a fait dans le passé sur amazon.com vont déterminer les suggestions de produits " à intérêt " qui lui sont faites à différents endroits du site ; l'historique de ses recherches sur google.com va influencer l'ordre des sites qui lui sont proposés lors d'une nouvelle requête' ${ }^{1}$.

Dans cet article, nous nous intéressons de manière théorique à ces médiations algorithmiques qui participent de manière subtile à la structuration des niveaux de visibilité et, par conséquent, des opportunités de faire et de savoir qui se présentent aux internautes.

\section{Socionumérisation}

6 Le point de départ de notre réflexion consiste à rappeler que la numérisation est un phénomène à multiples facettes. Pour souligner que la modélisation en forme numérique ne s'arrête pas aux documents, contenus et catalogues mais pénètre de plus en plus les pratiques sociales et politiques, Latham et Sassen (2005) ont introduit le concept de " socionumérisation " (sociodigitization). Il s'agit de rendre visible une tendance à formaliser en forme de programme informatique les connaissances, procédures, règles et manières de faire qui composent une pratique, par exemple la gestion d'une entreprise (SAP) ou le maintien d'un réseau d'amis (Facebook). Bien que cette formalisation ne soit jamais totale - les usages débordent toujours du cadre de l'outil - la socionumérisation a pour effet d'ancrer certains aspects d'une pratique dans le monde des machines où chaque entité, relation ou processus est représenté en forme de donnée ou algorithme. Toute pratique socionumérisée devient ainsi dans un certain sens pratique informationnelle, c'est-à-dire qu'elle intègre des "situations de production, de recherche, et de traitement de l'information » (Ihadjadene et Chaudiron, 2009, 1). Dans l'univers de la 
machine, les mécanismes élaborés dans un domaine se laissent souvent transférer vers un autre : une technique de personnalisation par exemple peut s'appliquer à n'importe quelle procédure de sélection, une méthode de visualisation peut produire des images à partir de données variées. On peut donc observer aujourd'hui un phénomène remarquable : l'application de techniques issues du domaine de la recherche et de l'analyse de l'information à des phénomènes dont on ne dirait peut-être pas qu'ils soient d'ordre " informationnel ", dans le but de les représenter aussi bien que d'intervenir sur leur fonctionnement.

7 Les méthodes employées pour analyser et rendre opérable les traces d'usage sont issues d'une longue histoire en recherche d'information (RI) et des champs annexes comme l'informatique décisionnelle ou la fouille de données. Depuis leurs débuts dans les années 50 et 60 , ces domaines sont confrontés à une problématique qui guette à degré variable toute l'informatique : pour faire entrer une réalité externe dans la machine, il faut la modéliser et ce processus de formalisation est à la fois sélectif et épistémologiquement "orienté ", c'est-à-dire basé sur des préconceptions conceptuelles et normatives (Agre, 1997). Dans le cadre de l'informatique décisionnelle par exemple, les indicateurs utilisés pour rendre lisible une situation ou tendance économique sont basés sur un choix de données " pertinentes » et intégrés dans un modèle plus ou moins détaillé du domaine en question. Bien que ces méthodes revendiquent une rationalité impeccable, il est clair qu'ils ne peuvent pas se détacher d'un fond normatif ; un modèle économique spécifique dessine et organise la réalité différemment d'autres modèles et les partis pris ont des conséquences réelles pour les réalités qu'ils se prêtent à décrire (MacKenzie, 2006). Le choix d'un modèle étant un acte potentiellement controversé, il prend une dimension politique.

8 L'écart entre une réalité sociale insoumise et les efforts pour la dompter intellectuellement aussi bien que dans les faits se creuse lorsqu'on regarde des terrains d'application plus " mous ». Prenons comme exemple les sites de rencontre, l'un des grands succès ${ }^{2}$ publics et commerciaux du Web, qui ne proposent rien d'autre que la socionumérisation du contact amoureux. Les chercheurs qui ont accès aux données produites à travers l'usage de ces sites - question problématique sur plusieurs niveaux - peuvent évidemment lancer des analyses tout à fait remarquables (Skopek et al., 2010), mais les outils intellectuels et techniques déployés pour faire parler ces données impliquent des choix conceptuels et méthodologiques qui vont orienter les représentations résultantes et, par conséquent, les connaissance produites. Or, sur le plan de l'intervention, la modélisation a des effets encore plus directs et les sites de rencontre montrent parfaitement comment la socionumérisation peut littéralement rendre opérable des modèles issus des SHS. Outre les médiations classiques par une interface qui structure les modes d'interaction, etc. cela implique une pénétration dans le cœur même de la question amoureuse. Dans le cadre de parship.fr, l'un des leaders du marché, certains choix de modélisation sont faits à partir de théories psychologiques qui cherchent à expliciter les facteurs qui contribuent à la réussite d'un couple. Le questionnaire de personnalité que tout nouveau inscrit doit remplir, « développé par des psychologues et scientifiques renommés » (Parship, 2010), prend en compte ces facteurs et a pour but de fournir des données exploitables dans le cadre d'un modèle décrivant le domaine, la vie de couple. Comme chaque usager produit ainsi un " double informationnel ", il n'est guère difficile de calculer, par le biais d'un " algorythme [sic] éprouvé " (ibid.), un " taux de compatibilité "(ibid.) entre tous les membres du site. Les chemins de 
navigation, les propositions par email et tous les résultats de recherche sont par la suite classés selon les résultats de cette application automatique d'un modèle psychologique. Implémenté en forme de logiciel, le modèle va donc intervenir sur l'espace social pour le préstructurer. voyons le passage d'un mode de fonctionnement basé sur un filtrage (éditorial) avant la publication (filter-then-publish) à une logique inverse où toutes les informations (textes, profils d'usagers, produits, etc.) sont en principe disponibles mais circonscrites par des couches de filtrage (publish-then-filter). Revenons à notre exemple : dans le monde hors ligne, les structures d'opportunité pour trouver un partenaire sexuel ou conjugal sont d'emblée encadrées par toute une série de distances, physiques et sociales. Avec une plateforme en ligne par contre le " marché " est en principe sans limites ; vous pouvez accéder à des millions de profils dans des dizaines de pays. Le premier filtrage se fait donc par un jeu de paramètres défini, médiatisé par un dispositif technique. La technicisation permet de traiter la surabondance de l'offre sociale comme un problème de surcharge informationnelle et, par conséquent, de le " résoudre " par des techniques issues de la RI.

Bien évidemment il ne s'agit pas d'une détermination par la technique des choix matrimoniaux ; mais la sélection des catégories composant le double informationnel de l'usager et des modèles algorithmiques de classement introduit des biais cognitifs (comment imaginer mon partenaire idéal ?) et pratiques (comment seront classés les résultats de ma recherche ?). Des études empiriques de grande taille (Skopek et al., 2010) confirment la tendance à l'homophilie (« qui se ressemble s'assemble ») sur les plateformes en ligne et identifient le niveau d'étude - élément qui se quantifie particulièrement bien - comme facteur dominant. Pour l'instant, le rôle spécifique du dispositif médiateur reste pourtant peu étudié. Or, tandis que la question de l'analyse des traces numériques comme méthode en SHS soulève des questions difficiles mais en partie connues de l'histoire de la statistique, la récursivité introduite par la médiation algorithmique pose de nouveaux problèmes. En introduisant le concept de " distance informationnelle " nous espérons établir une base pour des analyses ultérieures.

\section{Distances informationnelles, distances sociales}

11 La notion de distance informationnelle ${ }^{3}$ pourrait être définie comme la probabilité qu'un internaute se trouve confronté à une certaine information (un document, une idée, etc.). Pensée comme relationnelle, cette distance dépend à la fois de l'usager, de l'information et de l'ensemble des couches médiatrices. Dans un certain sens proche du « surfeur aléatoire $»^{4}$, avancé par les créateurs de Google dans leur présentation de PageRank (Brin et Page, 1998), le concept s'en distingue entre autre par une vision plus large de l'espace numérique en question. Car le Web n'est plus un espace topologique simple composé de pages statiques et de liens, il est de plus en plus sillonné par des systèmes hiérarchisant, filtrant et suggérant qui court-circuitent les chemins de navigation par lien. Plutôt que de censurer ou de dicter, ces systèmes modifient la distance qui sépare un utilisateur d'une information, distance que l'on pourrait exprimer, en termes simples, par le nombre de clicks qu'il faut pour la traverser. Mais une telle réduction risquerait de cacher le fait qu'afin de comprendre une pratique informationnelle dans sa profondeur, maints facteurs entrent en jeu, notamment le niveau de connaissance de l'usager et la 
disponibilité de chemins alternatifs qui pourraient passer par le travail éditorial ou le " bouche-à-oreille ». Le concept de distance informationnelle devrait donc permettre de penser ensemble dispositif technique et configuration sociale.

Curieusement, la sociologie dans la lignée de Simmel (1908) tente de rendre compte des phénomènes sociaux en termes d'une " géométrie sociale " où chaque individu se trouve à une certaine distance des autres. Dans les travaux de Rogers (1962), cette distance est définie simplement comme la probabilité que deux personnes communiquent ou interagissent. L'auteur va jusqu'à déduire la notion de " structure sociale » du fait que cette probabilité est évidemment distribuée de manière très inégale sur l'ensemble de la population ; les frontières spatiales, sociales, culturelles et économiques stratifient même les sociétés les plus homogènes. Le débat autour des " effets » des (N)TIC se divise justement sur cette question : l'utopie d'une société revigorée, cohésive et démocratisée (moins de distance sociale) s'oppose à la vision d'une société divisée, atomisée et désolidarisée (plus de distance sociale). Sans entrer dans ce débat, notre argument consiste à dire que les distances informationnelles peuvent jouer un rôle important dans le paramétrage des distances sociales. Volontairement ou non, les pratiques des usagers produisent des traces dont le traitement algorithmique risque d'avoir des conséquences pour leurs opportunités informationnelles et sociales, c'est-à-dire pour les contenus qu'ils trouvent et les contacts qu'ils établissent. Il convient de rajouter tout de suite que nous ne souscrivons pas à une causalité simple, à une détermination par la technique, mais à un horizon " probabiliste " où les objets pourraient " autoriser, rendre possible, mettre à portée, permettre, suggérer, influencer, faire obstacle, interdire, et ainsi de suite » (Latour, 2006, 103). Enfin, l'intégration de méthodes issues de la RI dans les mailles fines des espaces numériques peut encourager un " agir stratégique " à la manière des journalistes qui adaptent leur langage pour améliorer leur classement dans Google News. Comme l'a dit Simondon, " [l]a médiation entre l'homme et le monde devient elle-même un monde, la structure du monde " (Simondon, 1958, 181) et nous habitons cette structure de manière active.

Dans le but de " faciliter ", de rendre plus " efficace ", un grand nombre de services Web intègrent désormais des techniques de personnalisation, synthétisation, recommandation ou filtrage dont le fonctionnement est implicite (et souvent invisible), c'est-à-dire que l'usager n'a pas besoin de formuler des requêtes dans un champ de recherche pour être entouré de processus qui placent des liens, orientent la navigation, trient des listes, suggèrent des objets " chauds " ou " à intérêt ", filtrent, sélectionnent, mettent en perspective, cartographient, décident ${ }^{5}$. Nous baignons dans un milieu où la sélection des informations que nous consommons et des personnes avec qui nous interagissons est aussi le produit d'un traitement algorithmique orienté de représentations sélectives de nos gestes, nos idées et nos valeurs.

\section{Surveiller et gouverner}

Pour mieux comprendre les enjeux politiques de ce travail algorithmique, deux perspectives théoriques nous paraissent particulièrement pertinentes, dont chacune développe certains éléments de la pensée de Michel Foucault. La première se déploie à partir de la notion de surveillance, et s'intéresse notamment aux mécanismes disciplinaires qui cherchent à transformer les individus (potentiellement) désobéissants en « corps dociles ». Tandis que le versant « populaire " de cette approche perçoit 
les plateformes en ligne comme des extensions potentielles d'un appareil de police, un big brother politico-capitaliste, les surveillance studies anglo-saxonnes ont développé des concepts plus nuancés. Dans la suite de l'influent livre d'Oscar Gandy (1993), elles se sont notamment intéressées aux concepts de " triage » (sort), « the complex technology that involves the collection, processing, and sharing of information about individuals and groups that is generated through their daily lives as citizens, employees, and consumers and is used to coordinate and control their access to [...] goods and services [...] " (Gandy, 1993, 15). Dans les administrations publiques aussi bien que dans le secteur privé, l'usage et la mise en relation de multiples bases de données pour la classification et l'évaluation d'individus seraient en train de conduire à une " discrimination automatisée " (Lyon, 2002) séparant le client fiable de la faille potentielle et le citoyen honnête du perturbateur. Les systèmes qui captent nos gestes en ligne, pourraient-ils remplir une fonction d'exclusion et de normalisation similaire, que ce soit à travers des mécanismes de représentation ou d'intervention ? Nous pouvons certainement constater que la quantité des informations collectées et la puissance des moyens de les traiter augmentent rapidement, " renforçant le pouvoir social de l'information " (Lyon, 2002, 22) et créant un outil de pouvoir qui reste pour l'instant peu encadré.

Une deuxième perspective peut être développée à partir des derniers travaux de Foucault, notamment des cours donnés au Collège de France dans lesquels sont introduits différents éléments d'un mécanisme de pouvoir, nommé la sécurité (Foucault, 2004a), qui se distingue de la logique de la discipline. Ce concept s'intègre dans l'interrogation sur la " gouvernementalité ", la " manière dont on conduit la conduite des hommes " (Foucault, 2004b, 192), que Foucault développe en articulation avec une analyse du libéralisme contemporain. Celui-ci serait surtout caractérisé par l'instauration du marché ${ }^{6}$ comme lieu de vérité où le libre jeu de forces produit le " juste prix ", le " vrai prix » (ibid., 33). Le " bon art de gouverner " consisterait donc à sauvegarder contre les interventions externes (l'État) et les concentrations internes (le monopole). La sécurité comme mécanisme de pouvoir va alors moins viser le comportement des individus, comme l'avait fait la discipline, mais plutôt veiller sur le paramétrage d'une entité abstraite corrélative au marché, la population. Se trouvent donc au centre de la sécurité les sciences sociales et notamment la statistique, des outils de connaissance permettent de gouverner par des ajustements subtils des règles du marché. Sur le plan de la représentation, les différentes méthodes d'analyse des traces informationnelles s'intègrent parfaitement dans cet arsenal et accomplissent, à l'image de Google Trends, une extension vers le quasi temps réel. Pour le marketing, ces plateformes constituent, dans un sens très littéral, des lieux de vérité, le jeu des désirs définissant les besoins et les opportunités économiques. Sur le plan de l'intervention, la structuration des opportunités de faire et de savoir introduit un outil de gestion redoutable, bien qu'il faille le comprendre plus dans une logique de déblocage que de contrôle. Le régime de sécurité n'est pas un mécanisme disciplinaire qui vise la modélisation d'une société selon une vérité universelle, qu'elle soit spirituelle ou morale ; c'est un mécanisme de gouvernance qui cherche à connaître et à organiser la société par l'agrégation et l'analyse d'une infinité de microdécisions prises par sa population. Au lieu de discriminer et de trier, l'enjeu consiste à gouverner les sujets à travers leur liberté en tant qu'acteurs dans un marché généralisé. Dans une telle perspective, l'analyse des traces numériques joue un double rôle : elle fournit les outils techniques et intellectuels pour cartographier le fourmillement dans les espaces en ligne (représentation) et elle garantit le fonctionnement même de ces espaces 
en introduisant des fonctions " cognitives " - filtrer, suggérer, synthétiser, etc. sans lesquelles la masse des informations et acteurs devient rapidement écrasante ( intervention). Sa première fonction politique serait donc de permettre l'extension d'une certaine manière de comprendre et de gouverner la société.

\section{Conclusions}

Pour conclure notre argument, nous voudrions souligner trois éléments :

Premièrement, dans une perspective épistémologique (dans le sens kantien, " théorie de la connaissance "), l'introduction de mécanismes statistiques et autres dans de nombreux endroits $\mathrm{du}$ Web est étroitement liée à une manière de " parler vrai " qui se veut empirique plutôt qu'axiomatique, déductive plutôt qu'inductive. Au lieu de développer des chaines de raisonnement, elle cherche à " laisser parler les chiffres ", ou, de plus en plus, les cartes et graphes. Cette production de connaissance procède par l'agrégation et l'analyse des traces informationnelles qui, pour reprendre Tarde, "s'échappent de chaque fait social ». Cela ne nous paraît ni bon, ni mauvais en soi ; mais il est urgent de débattre les enjeux d'un tel développement.

Deuxièmement, les structures de données et algorithmes jouent un rôle pour les techniques du pouvoir, pour la « manière dont on conduit la conduite des hommes ». La socionumérisation réorganise les roues et leviers de notre très grande machine sociotechnique et le contrôle démocratique de ces transformations est loin d'être assuré. La question du traitement des traces des pratiques en ligne comme source de structuration était au centre de notre interrogation mas qu'en est-il de la traçabilité du traçage ? Comment examiner et contrôler des techniques en boîte noire, protégés à la fois par le fonctionnement de l'espace numérique (sans code source, il est très difficile d'analyser un algorithme) et de l'espace juridique (le secret professionnel). La question de savoir comment étudier ces objets est donc elle même un enjeu politique.

Troisièmement, il se pose une véritable question de déontologie. Si nous participons - en tant que chercheurs, ingénieurs, développeurs et concepteurs - à la structuration du jeu des distances et proximités, comment formuler des valeurs et règles de conduite ? Est-ce qu'il suffit de parler des " besoins » des usagers ou faudrait-il commencer à penser " la société en tant que client " (Simon, 1969)?

17 Sur ces trois points, nous n'avons pas de réponses toutes faites à proposer, mais nous sommes persuadés que l'avancement de la socionumérisation rend nécessaire une analyse plus conséquente de la dimension politique des objets et environnements techniques. Cette tâche nécessite une mise en question de ce que nous entendons par " technique » aussi bien que par « politique ».

\section{BIBLIOGRAPHIE}

Agre, P., (1997), Computation and human experience, Cambridge, Cambridge University Press, 392 p. 
Brin, S. et Page, L., (1998), The anatomy of a large-scale hypertextual Web search engine, http:// infolab.stanford.edu/ backrub/google.html, date de la dernière visite : 22 avril 2010.

Foucault, M., (2004a), Sécurité, territoire, population : Cours au Collège de France (1977-1978), Paris, Gallimard/Seuil, 435 p.

Foucault, M., (2004b), Naissance de la biopolitique : Cours au Collège de France (1978-1979), Paris, Gallimard/Seuil, 355 p.

Gandy, O. H., (1993), The panoptic sort : a political economy of personal information, Boulder/Oxford, Westview, 283 p.

Hacking, I., (1983), Representing and intervening : introductory topics in the philosophy of natural science, Cambridge, Cambridge University Press, 302 p.

Ihadjadene, M. et Chaudiron, S., (2009), Des processus aux pratiques : quels modèles informationnels pour analyser l'accès à l'information en contexte professionnel ?, http://hal.archives-ouvertes.fr/ hal-00468728/, date de la dernière visite : 10 août 2010.

Latham, R. et Sassen, S., (2005), Digital formations : IT and new architectures in the global realm, Princeton, Princeton University Press, $367 \mathrm{p}$.

Latour, B., (2007), Changer de société, refaire de la sociologie, Paris, La Découverte, 401 p.

Lyon, D., (2002), Surveillance as social sorting : privacy, risk and automated discrimination, London/ New York, Routledge, 287 p.

MacKenzie, D., (2006), An engine, not a camera : how financial models shape markets, Cambridge MA, MIT Press, 377 p.

Metz, C., (2010), Google «personalizes » one in five searches. Eye on your history, your location, your friends, http://www.theregister.co.uk/2010/03/03/google_personalized_search_explained/, date de la dernière visite : 22 avril 2010.

O’Reilly, T. et Battelle, J., (2009), Web squared : Web 2.0 five years on, http:// www.web2summit.com/web2009/public/schedule/detail/10194, date de la dernière visite : 22 avril 2010.

Parship, (2010), Comment ça marche, http://www.parship.fr/editorial/this_is_parship/ how_it_works/index.html, date de la dernière visite : 22 avril 2010.

Rogers, E. M., (1962, 2003), Diffusion of innovations, fifth edition, New York, Free Press, 551 p.

Shirky, C., (2008), Here comes everybody : the power of organizing without organizations, London, Penguin, $327 \mathrm{p}$.

Simmel, G., (1908), Untersuchungen über die Formen der Vergesellschaftung, Berlin, Duncker \& Humblot, $782 \mathrm{p}$.

Simon, H. A., (1969), The Sciences of the Artificial, Cambridge MA, MIT Press, 123 p.

Simondon, G., (1958), Du mode d'existence des objets techniques, Paris, Aubier, 333 p.

Skopek, J., Schulz, F. et Blossfeld, H.-P., (2010), Who contacts whom ? Educational homophily in online mate selection, European Sociological Review Advance Access, DOI:10.1093/esr/jcp068.

Tarde, G., (1890, 2001), Les Lois de l'imitation, Paris, Seuil, 445 p. 


\section{NOTES}

1. Google Search intègre aujourd'hui trois axes de personnalisation (Metz 2010) : l'historique des recherches d'un utilisateur, sa localité et les contacts enregistrés dans son carnet de Gmail ou Google Chat.

2. Selon ses propres affirmations, meetic, l'un des leaders du marché avait fin décembre 2009, 850.000 abonnés dans 16 pays, payant chacun en moyenne $18 €$ par mois, (http://www.meeticcorp.com/fr/metrics.html).

3. Notons que notre concept de «distance informationnelle» se distingue de celui développé dans le cadre d'une théorie mathématique de l'information, notamment par Charles H. Bennett. Nous n'essayons pas de formuler un modèle formel ou quantitatif mais de trouver une voie pour penser en parallèle structures techniques et sociales.

4. Brin et Page avancent plusieurs raisonnements pour justifier PageRank, dont le modèle d'un internaute qui suit de manière aléatoire les liens qui se présentent devant lui. La probabilité de tomber sur un site particulier est proportionnelle à la connexité de ce site dans le réseau de liens et PageRank n'est rien d'autre que l'application de cette mesure de distance topologique au classement des résultats d'une recherche.

5. Les derniers efforts de Facebook pour étendre sa plateforme sur le Web entier représentent un bon exemple. Depuis avril 2010, n'importe quel site peut proposer une offre personnalisée à ses visiteurs en accédant à certaines informations de leurs profils Facebook via un service Web (API), sans avoir besoin que l'usager se connecte explicitement.

6. L'auteur se sent obligé d'avertir le lecteur: notre usage du terme "marché » se veut strictement neutre. Nous n'y associons, ici, aucune volonté de « dénonciation ».

\section{RÉSUMÉS}

La croissance des espaces numériques et l'infiltration des NTIC dans un nombre important de pratiques quotidiennes conduisent à la production de toujours plus de traces numériques. Ces traces sont analysées par le marketing et la recherche mais elles servent aussi à filtrer et classer les résultats d'une recherche, les options de navigation proposées ou, sur les sites sociaux, le choix des personnes à rencontrer. Dans ce texte, nous examinons l'analyse des traces numériques comme mode de représentation et outil d'intervention, d'organisation et de gouvernance.

The growth of digital spaces and the infiltration of ICT into a large number of everyday practices lead to an increasing production of digital traces. These traces are being analyzed by marketers and researchers but they are also used to filter and rank search results, possibilities for navigation, and - on social sites - the choice of people to meet. In this text we examine the analysis of digital traces as a mode of representation as well as a tool for intervention, organization, and governance. 


\section{INDEX}

Keywords : analysis of digital traces, digital politics, online sociability, power, statistical method, information seeking and use

Mots-clés : analyse des traces numériques, médiations algorithmiques, politique du numérique, sociabilité en ligne, pouvoir, méthode statistique, usage de l'information, recherche

d'information

\section{AUTEUR}

\section{BERNHARD RIEDER}

Laboratoire Paragraphe - Université de Paris VIII

Bernhard Rieder est membre du laboratoire paragraphe de l'Université Paris 8. Son travail porte principalement sur le rapport entre technique et société, en ce qui concerne la médiation des pratiques sociales et la production de connaissances par les outils numériques aussi bien que la question de la conception des systèmes informatiques comme pratique politique. Adresse électronique : bernhard.rieder@univ-paris8.fr. 